\title{
FRT PRESENTATION OF THE ONSAGER ALGEBRAS
}

\author{
PASCAL BASEILHAC*, SAMUEL BELLIARD**, AND NICOLAS CRAMPÉ ${ }^{\dagger, *}$
}

\begin{abstract}
A presentation à la Faddeev-Reshetikhin-Takhtajan (FRT) of the Onsager, augmented Onsager and $s l_{2}$-invariant Onsager algebras is given, using the framework of the non-standard classical Yang-Baxter algebras. Associated current algebras are identified, and generating functions of mutually commuting quantities are obtained.
\end{abstract}

MSC: 81R50; 81R10; 81U15.

Keywords: Onsager algebras; Tridiagonal algebra; Current algebra; Yang-Baxter algebra; Integrable systems.

\section{INTRODUCTION}

Introduced by L. Onsager in the investigation of the exact solution of the two-dimensional Ising model O44, the Onsager algebra is an infinite dimensional Lie algebra with two known presentations. The original presentation is given in terms of generators $\left\{A_{n}, G_{m} \mid n, m \in \mathbb{Z}\right\}$ and relations (see Definition 4.1). A second presentation ${ }^{1}$ is given in terms of two generators $A_{0}, A_{1}$ satisfying the so-called Dolan-Grady relations (4.5) DG82. Later on, it was shown that the Onsager algebra is isomorphic to a fixed point subalgebra of the affine Kac-Moody algebra $\widehat{s l_{2}}$ under the Chevalley involution [D91, R91].

In the context of mathematics and quantum integrable systems, a $q$-deformed analog of the Onsager algebra has been introduced in recent years [T99, B04. It is isomorphic to a coideal subalgebra of $U_{q}\left(\widehat{s l_{2}}\right)$ [BB09] (see also [K12]). Similarly to the classical (undeformed) Onsager algebra, two presentations are known, which can be viewed as $q$-deformed analog of the original presentations of Onsager BK05, BK17. and Dolan-Grady [T99, B04. Other types of algebras have been later on considered in the literature: the augmented $(q-)$ Onsager algebra [TT09, BB12, BC12] and the $U_{q}\left(g l_{2}\right)$-invariant $q$-Onsager algebra [BB17]. Given the known automorphisms of $U_{q}\left(\widehat{s l_{2}}\right)$, these other types of $q$-Onsager algebras can be understood as different coideal subalgebras of $U_{q}\left(\widehat{s l_{2}}\right)$. For all these different types of $q$-Onsager algebras, a third presentation is also identified [B04, BS09, BB17, using the framework of the quantum reflection algebra [S88. Based on this presentation, one obtains current algebras for the $q$-Onsager algebras [BS09] that found applications in the solution of the open XXZ spin chain BB12. This third presentation gives also a tool to derive explicit examples of tridiagonal pairs [T99] or to conjecture a Poincaré-Birkhoff-Witt basis for the $q$-Onsager algebra [BB17, besides the so-called zig-zag basis of Ito-Terwilliger [IT09].

By analogy with the family of $q$-Onsager algebras that admit a presentation within the framework of the quantum reflection algebra, it is thus natural to search for this 'missing' presentation of the classical Onsager algebras. In this letter, we answer this question. Namely, it is shown that all classical Onsager algebras admit a presentation within the framework of the non-standard classical Yang-Baxter algebras. This link offers a new perspective to study the related integrable systems.

This letter is organized as follows. In Section 2, the framework of the classical Yang-Baxter algebra $\mathscr{T}$ (with central extension) and the non-standard classical Yang-Baxter algebra $\mathscr{B}$ is settled. Automorphisms of $\mathscr{T}$ are considered, which are used to construct homomorphisms from $\mathscr{B}$ to $\mathscr{T}$. Also, based on solutions of the classical reflection equation, a different homomorphism is proposed. Then, two commutative subalgebras of $\mathscr{B}$ are identified. In Section 3, we construct some of the simplest explicit examples of non-standard classical Yang-Baxter algebras $\mathscr{B}$ related with the affine Kac-Moody algebra $\widehat{s l_{2}}$. First, we introduce some necessary material. Different known presentations of $\widehat{s l_{2}}$ are also recalled: the Serre-Chevalley presentation [K85, the Cartan-Weyl presentation [GO86] and the so-called FRT presentation (in honour of FaddeevReshetikhin-Takthadjan [FRT87]). As an application of the results of Section 2, it is shown that known

\footnotetext{
${ }^{1}$ The connection between the Dolan-Grady construction and the original Onsager algebra was understood by J.H.H. Perk in 1982 P87.
} 
automorphisms of $\widehat{s l_{2}}$ are easily recovered from the automorphisms of the classical Yang-Baxter algebra. Then, three different non-standard classical Yang-Baxter algebras are considered. In each case, the map to subalgebras of $\widehat{{s l_{2}}_{2}}$ is described in details. In Section 4, it is shown that these three non-standard classical Yang-Baxter algebras are isomorphic to the Onsager algebra, the augmented Onsager algebra and the $s l_{2}$ invariant Onsager algebra, respectively. In each case, our construction provides an FRT presentation. Their corresponding current presentations are displayed. Finally, as an application of the FRT presentations, we derive generating functions of mutually commuting quantities for each of the Onsager algebras. Concluding remarks are given in Section 5.

\section{Classical Yang-Baxter algebras and commutative subalgebras}

In this section, we introduce the basic definitions of the standard and non-standard classical Yang-baxter algebras, denoted $\mathscr{T}$ and $\mathscr{B}$, respectively. See Definitions 2.2 and 2.4, A class of automorphisms of $\mathscr{T}$ is exhibited in Proposition 2.1, and the homomorphic image of $\mathscr{B}$ into the fixed point subalgebra of $\mathscr{T}$ under this automorphism is described in Proposition 2.2. A different homomorphic image of $\mathscr{B}$ into a subalgebra of $\mathscr{T}$ is also given in Proposition 2.3, based on solutions of the classical reflection equation. Finally, two different commutative subalgebras of $\mathscr{B}$ are displayed, see Propositions 2.4 and 2.5 .

2.1. Classical Yang-Baxter algebras. The classical Yang-Baxter equation appeared as a limit of the quantum inverse scattering method [S80] and became quickly popular [BD82, D83, S83. In this letter, we use two slightly different definitions (see Definitions 2.1 and 2.3).

Definition 2.1. The matrix $r(x) \in \operatorname{End}\left(\mathbb{C}^{N} \otimes \mathbb{C}^{N}\right)$ is called a classical r-matrix if it satisfies the skewsymmetric condition $r_{12}(x)=-r_{21}(1 / x)$ and the classical Yang-Baxter equation

$$
\left[r_{13}\left(x_{1} / x_{3}\right), r_{23}\left(x_{2} / x_{3}\right)\right]=\left[r_{13}\left(x_{1} / x_{3}\right)+r_{23}\left(x_{2} / x_{3}\right), r_{12}\left(x_{1} / x_{2}\right)\right]
$$

for any $x_{1}, x_{2}, x_{3}$. Here, $r_{12}(x)=r(x) \otimes \mathbb{I}, r_{23}(x)=\mathbb{I} \otimes r(x)$ and so on.

For a given skew-symmetric r-matrix $r(x)$ that satisfies the classical Yang-Baxter equation, we now introduce the Lie algebra $\mathscr{T}$ with central extension $c$. Generically, this algebra is called the classical Yang-Baxter algebra. Note that this algebra can be understood as a classical analog of the algebra introduced in [RS90.

Let $\left\{E_{i j} \mid 1 \leq i, j \leq N\right\}$ denotes the standard basis of $\operatorname{End}\left(\mathbb{C}^{N}\right)$ (i.e. the $N \times N$ matrices with components $\left.\left(E_{i j}\right)_{k l}=\delta_{i k} \delta_{j l}\right)$. Below we use the Einstein summation convention by omitting the sums over the repeated indices $i, j$.

Definition 2.2. Let $r(x) \in \operatorname{End}\left(\mathbb{C}^{N} \otimes \mathbb{C}^{N}\right)$ be a classical $r$-matrix. $\mathscr{T}$ is the Lie algebra with generator $2^{2}$ $\left\{c, t_{i j}^{ \pm[\ell]} \mid 1 \leq i, j \leq N, \ell \in \mathbb{Z}\right\}$. Introduce:

$$
T^{+}(x)=E_{i j} \otimes \sum_{\ell \in \mathbb{Z}} t_{i j}^{+[\ell]} x^{\ell}, \quad T^{-}(x)=E_{i j} \otimes \sum_{\ell \in \mathbb{Z}} t_{i j}^{-[\ell]} x^{\ell} .
$$

The defining relations are:

$$
\begin{aligned}
{\left[T^{ \pm}(x), c\right] } & =0 \\
{\left[T_{1}^{ \pm}(x), T_{2}^{ \pm}(y)\right] } & =\left[T_{1}^{ \pm}(x)+T_{2}^{ \pm}(y), r_{12}(x / y)\right], \\
{\left[T_{1}^{+}(x), T_{2}^{-}(y)\right] } & =\left[T_{1}^{+}(x)+T_{2}^{-}(y), r_{12}(x / y)\right]-2 c r_{12}^{\prime}(x / y) x / y .
\end{aligned}
$$

Remark 1. The Jacobi identity of the Yang-Baxter algebra $\mathscr{T}$ follows from the classical Yang-Baxter equation and its derivative. The latter reads:

$$
\left[f_{13}\left(x_{1} / x_{3}\right)+f_{23}\left(x_{2} / x_{3}\right), r_{12}\left(x_{1} / x_{2}\right)\right]=\left[f_{13}\left(x_{1} / x_{3}\right), r_{23}\left(x_{2} / x_{3}\right)\right]+\left[r_{13}\left(x_{1} / x_{3}\right), f_{23}\left(x_{2} / x_{3}\right)\right]
$$

where $f(x)=x r^{\prime}(x)$.

The presentation of the commutation relations (2.3)-(2.5) of a Lie algebra $\mathscr{T}$ is called a FRT presentation in honour of the authors Faddeev-Reshetikhin-Takhtajan [FRT87.

A class of automorphisms of the Lie algebra $\mathscr{T}$ is now considered.

\footnotetext{
${ }^{2}$ According to the examples, the range of $\ell$ will be restricted.
} 
Proposition 2.1. Let $r(x)$ be a classical $r$-matrix with the additional property $r_{12}(x)=-r_{12}(1 / x)^{t_{1} t_{2}}$ and $U(x)$ be a $N \times N$ invertible matrix satisfying

$$
U(x)^{t}=\epsilon U(1 / x) \quad \text { and } \quad U_{1}(x) U_{2}(y) r_{12}(x / y)=r_{12}(x / y) U_{1}(x) U_{2}(y) \quad \text { for } \epsilon=+1 \text { or } \epsilon=-1 .
$$

Then, the map $\theta: \operatorname{End}\left(\mathbb{C}^{N}\right) \otimes \mathscr{T} \rightarrow \operatorname{End}\left(\mathbb{C}^{N}\right) \otimes \mathscr{T}$ such that

$$
T^{ \pm}(x) \mapsto U(x) T^{\mp}(1 / x)^{t} U(x)^{-1} \mp c x U^{\prime}(x) U(x)^{-1}, \quad c \mapsto-c,
$$

where $(.)^{t}$ stands for the transposition in End $\left(\mathbb{C}^{N}\right)$, provides an involutive automorphism of $\mathscr{T}$.

Proof. First, we show that $\theta$ is an automorphism. The action of $\theta$ on (2.3) is trivially computed. Consider the action of $\theta$ on (2.4). After straightforward simplifications, the corresponding equation reduces to:

$$
\left[x U_{1}^{\prime}(x) U_{1}(x)^{-1}+y U_{2}^{\prime}(y) U_{2}(y)^{-1}, r_{12}(x / y)\right]=0 .
$$

Similarly, consider the action of $\theta$ on (2.5). The corresponding equation reduces to:

$$
\begin{aligned}
& 2 \frac{x}{y}\left(U_{1}(x) U_{2}(y) r_{12}^{\prime}(x / y) U_{1}(x)^{-1} U_{2}(y)^{-1}-r_{12}^{\prime}(x / y)\right) \\
& +\left[x U_{1}^{\prime}(x) U_{1}(x)^{-1}-y U_{2}^{\prime}(y) U_{2}(y)^{-1}, r_{12}(x / y)\right]=0 .
\end{aligned}
$$

It is easy to show that these last two equations follow from linear combinations of the derivatives of (2.6) either with respect to $x d / d x$ or with respect to $y d / d y$. It remains to show the involution property. One has:

$$
\begin{aligned}
\theta^{2}\left(T^{+}(x)\right)= & U(x)\left(\theta\left(T^{-}(1 / x)\right)\right)^{t} U(x)^{-1}+c x U^{\prime}(x) U(x)^{-1} \\
= & U(x)\left(U(1 / x) T^{+}(x)^{t} U(1 / x)^{-1}\right)^{t} U(x)^{-1}+c x U^{\prime}(x) U(x)^{-1} \\
& +c \frac{1}{x} U(x)\left(U^{\prime}(1 / x) U^{-1}(1 / x)\right)^{t} U(x)^{-1} .
\end{aligned}
$$

Then, we use the first equation of (2.6) and its derivative with respect to $x d / d x$, which gives $U^{\prime}(x)=$ $-\epsilon U^{\prime}(1 / x)^{t} / x^{2}$. The equation above simplifies to $\theta^{2}\left(T^{+}(x)\right)=T^{+}(x)$. We show similarly $\theta^{2}\left(T^{-}(x)\right)=T^{-}(x)$ (and $\theta^{2}(c)=c$ ), which concludes the proof.

2.2. Non-standard classical Yang-Baxter equation and the algebra $\mathscr{B}$. The non-standard classical Yang-Baxter equation can be understood as a generalization of the classical Yang-Baxter equation (2.1).

Definition 2.3. The matrix $\bar{r}(x, y) \in \operatorname{End}\left(\mathbb{C}^{N} \otimes \mathbb{C}^{N}\right)$ is called a non-standard classical r-matrix if it is a solution of the non-standard classical Yang-Baxter equation

$$
\left[\bar{r}_{13}\left(x_{1}, x_{3}\right), \bar{r}_{23}\left(x_{2}, x_{3}\right)\right]=\left[\bar{r}_{21}\left(x_{2}, x_{1}\right), \bar{r}_{13}\left(x_{1}, x_{3}\right)\right]+\left[\bar{r}_{23}\left(x_{2}, x_{3}\right), \bar{r}_{12}\left(x_{1}, x_{2}\right)\right]
$$

for any $x_{1}, x_{2}, x_{3}$.

In the case where the non-standard classical r-matrix depends on only one parameter $\bar{r}_{12}(x, y)=r_{12}(x / y)$ and is skew-symmetric $\bar{r}_{12}(x, y)=-\bar{r}_{21}(y, x)$ then the non-standard classical Yang-Baxter equation reduces to the standard one (2.1).

For a given r-matrix $\bar{r}(x, y)$ that satisfies the non-standard classical Yang-Baxter equation, we now introduce the Lie algebra $\mathscr{B}$.

Definition 2.4. $\mathscr{B}$ is the Lie algebra with generators $\left\{b_{i j}^{[\ell]} \mid 1 \leq i, j \leq N, \ell \in \mathbb{Z}\right\}$. Introduce:

$$
B(x)=E_{i j} \otimes \sum_{\ell \in \mathbb{Z}} b_{i j}^{[\ell]} x^{\ell} .
$$

The defining relations are:

$$
\left[B_{1}(x), B_{2}(y)\right]=\left[\bar{r}_{21}(y, x), B_{1}(x)\right]+\left[B_{2}(y), \bar{r}_{12}(x, y)\right],
$$

where $\bar{r}(x, y)$ satisfies the non-standard classical Yang-Baxter equation (2.9). 
Note that the Jacobi identity for $\mathscr{B}$ is guaranteed by the fact that the classical r-matrix in (2.11) satisfies the non-standard classical Yang-Baxter equation (2.9). Although this r-matrix is not in general skewsymmetric the Lie bracket of $\mathscr{B}$ is still anticommutative.

Given an automorphism $\theta$ of $\mathscr{T}$, a realization of the algebra $\mathscr{B}$ into a fixed point subalgebra $\sqrt{3}$ of $\mathscr{T}$ is now considered.

Proposition 2.2. Assume $r_{12}(x)=-r_{12}(1 / x)^{t_{1} t_{2}}, U(x)$ is a solution of (2.6) and $\theta$ is defined by (2.7). The map $\psi_{\theta}: \mathscr{B} \rightarrow \mathscr{T}$ such that

$$
B(x) \mapsto T^{+}(x)+\theta\left(T^{+}(x)\right)=T^{+}(x)+U(x) T^{-}(1 / x)^{t} U(x)^{-1}-c x U^{\prime}(x) U(x)^{-1},
$$

with

$$
\bar{r}_{12}(x, y)=r_{12}(x / y)+U_{1}(x) r_{12}^{t_{1}}(1 /(x y)) U_{1}(x)^{-1}
$$

is an algebra homomorphism.

Proof. See the proof of the Proposition 2.3 which is more general according to the Remark 2

In the following, we denote by $\mathscr{T}^{\theta} \subset \mathscr{T}$ the image of the algebra $\mathcal{B}$ by the homomorphism $\psi_{\theta}$. Let us remark that in the previous proposition, we may have considered $B(x) \mapsto T^{-}(x)+\theta\left(T^{-}(x)\right)$. However, it defines an isomorphic subalgebra.

To conclude this Section, we would like to point out that other subalgebras of $\mathscr{T}$, that are not necessarily fixed point subalgebras, may be considered as well. Indeed, along the lines of [Sk06b], let us consider a special case of the classical reflection equation [S87, eq. (2)].

Definition 2.5. The matrix $k(x) \in \operatorname{End}\left(\mathbb{C}^{N}\right)$ is called a $k$-matrix if it is a solution of the following equation

$$
r_{12}(x / y) k_{1}(x) k_{2}(y)-k_{1}(x) k_{2}(y) r_{12}(x / y)=k_{1}(x) r_{12}^{t_{2}}(x y) k_{2}(y)-k_{2}(y) r_{12}^{t_{2}}(x y) k_{1}(x)
$$

for a given skew-symmetric $r$-matrix $r(x)$ satisfying $r_{12}(x)=-r_{12}(1 / x)^{t_{1} t_{2}}$.

Remark 2. The matrices $U(x)$ solving (2.6) are examples of $k$-matrices.

We are now in position to give a generalization of Proposition 2.2 .

Proposition 2.3. For a given k-matrix $k(x)$, the map $\mathscr{B} \rightarrow \mathscr{T}$ such that

$$
B(x) \mapsto T^{+}(x)+k(x) T^{-}(1 / x)^{t} k(x)^{-1}-c x k^{\prime}(x) k(x)^{-1},
$$

with

$$
\bar{r}_{12}(x, y)=r_{12}(x / y)+k_{1}(x) r_{12}^{t_{1}}(1 /(x y)) k_{1}(x)^{-1}
$$

is an algebra homomorphism.

Proof. Inserting (2.15) into (2.11) and using (2.4), (2.5), one identifies $\bar{r}_{12}(x, y)$ as (2.16). Also, one finds a term in $c$ with coefficient:

$$
\begin{array}{ccc}
2 x y\left(k_{1}(x) r_{12}^{\prime t_{2}}(x y) k_{2}(y)-k_{2}(y) r_{12}^{\prime t_{2}}(x y) k_{1}(x)\right) & -x\left[r_{12}(x / y)+k_{2}(y) r_{12}^{t_{2}}(x y) k_{2}(y)^{-1}, k_{1}^{\prime}(x) k_{1}(x)^{-1}\right] k_{1}(x) k_{2}(y) \\
(2.17) & +y\left[k_{2}^{\prime}(x) k_{2}(x)^{-1}, r_{12}(x / y)-k_{1}(x) r_{12}^{t_{2}}(x y) k_{1}(y)^{-1}\right] k_{1}(x) k_{2}(y) .
\end{array}
$$

To show that this coefficient vanishs, we use the reflection equation (2.14) from which we deduce:

$$
\begin{aligned}
& k_{1}(x)^{-1} r_{12}(x / y) k_{1}(x) k_{2}(y)+k_{1}(x)^{-1} k_{2}(y) r_{12}^{t_{2}}(x y) k_{1}(x)=r_{12}^{t_{2}}(x y) k_{2}(y)+k_{2}(y) r_{12}(x / y), \\
& k_{2}(y)^{-1} r_{12}(x / y) k_{1}(x) k_{2}(y)-k_{1}(x) k_{2}(y)^{-1} r_{12}^{t_{2}}(x y) k_{2}(y)=k_{1}(x) r_{12}(x / y)-r_{12}^{t_{2}}(x y) k_{1}(x) .
\end{aligned}
$$

Using these two equations, the condition on the coefficient (2.17) yields to the constraint:

$$
\begin{aligned}
{\left[r_{12}(x / y), x k_{1}^{\prime}(x) k_{2}(y)+y k_{1}(x) k_{2}^{\prime}(y)\right] } & -x k_{1}^{\prime}(x) r_{12}^{t_{2}}(x y) k_{2}(y)-y k_{1}(x) r_{12}^{t_{2}}(x y) k_{2}^{\prime}(y) \\
& +x k_{2}(y) r_{12}^{t_{2}}(x y) k_{1}^{\prime}(x)+y k_{2}^{\prime}(y) r_{12}^{t_{2}}(x y) k_{1}(x) \\
& -2 x y\left(k_{1}(x) r_{12}^{\prime t_{2}}(x y) k_{2}(y)-k_{2}(y) r_{12}^{\prime t_{2}}(x y) k_{1}(x)\right)=0 .
\end{aligned}
$$

Taking linear combinations of the derivatives of (2.14) either with respect to $x d / d x$ or with respect to $y d / d y$, one finds that this constraint is satisfied.

\footnotetext{
${ }^{3}$ Also called twisted algebra, where the twist is the automorphism.
} 
Note that the r-matrix defined by (2.16) is a solution of the non-standard classical Yang-Baxter equation (2.9), as shown in Sk06a].

The homomorphic image of $\mathscr{B}$ defined in Proposition 2.3 is a Lie subalgebra of $\mathscr{T}$ that is not necessarily a fixed point subalgebra. It is denoted by $\mathscr{T}^{k}$.

2.3. Commutative subalgebras. Two commutative subalgebras of $\mathscr{B}$ are now identified. The first subalgebra has already appeared in the literature. The second subalgebra, which is new, will be of interest in the analysis of further sections.

The following proposition has been proven in [J89, HKW92, H95] to prove the integrability of the Gaudin models G83. and has been generalized in [CRT04.

Proposition 2.4. The following generating function in the universal enveloping algebra of $\mathscr{B}$

$$
t(x)=\operatorname{tr}\left(B(x)^{2}\right)=\sum_{\ell, p \in \mathbb{Z}} b_{i j}^{[\ell]} b_{j i}^{[p]} x^{\ell+p}
$$

satisfies

$$
[t(x), t(y)]=0
$$

Proposition 2.5. Let $\bar{r}(x, y)$ be a solution of the non-standard classical Yang-Baxter equation (2.9). Let $M(x) \in \operatorname{End}\left(\mathbb{C}^{N}\right)$ be a solution of the following equation

$$
\left[\operatorname{tr}_{1}\left(\bar{r}_{12}(x, y) M_{1}(x)\right), M_{2}(y)\right]=0 .
$$

Then,

$$
b(x)=\operatorname{tr} M(x) B(x)
$$

satisfies

$$
[b(x), b(y)]=0 .
$$

Proof. Multiply (2.11) on the left by $M_{1}(x) M_{2}(y)$ and take the traces in the spaces 1 and 2 to get

$$
\begin{aligned}
{[b(x), b(y)]=} & \operatorname{tr}_{12} M_{1}(x) M_{2}(y)\left(\bar{r}_{21}(y, x) B_{1}(x)-B_{1}(x) \bar{r}_{21}(y, x)\right) \\
& +t_{12} M_{1}(x) M_{2}(y)\left(B_{2}(y) \bar{r}_{12}(x, y)-\bar{r}_{12}(x, y) B_{2}(y)\right) \\
= & \left.t_{1} M_{1}(x) \operatorname{tr}_{2}\left(M_{2}(y) \bar{r}_{21}(y, x)\right) B_{1}(x)-\operatorname{tr}_{1} \operatorname{tr}_{2}\left(\bar{r}_{21}(y, x) M_{2}(y)\right) M_{1}(x) B_{1}(x)\right) \\
& +t_{2} \operatorname{tr}_{1}\left(\bar{r}_{12}(x, y) M_{1}(x)\right) M_{2}(y) B_{2}(y)-\operatorname{tr}_{2} M_{2}(y) \operatorname{tr}_{1}\left(M_{1}(x) \bar{r}_{12}(x, y)\right) B_{2}(y) \\
= & \operatorname{tr}_{1}\left[M_{1}(x), \operatorname{tr}_{2}\left(M_{2}(y) \bar{r}_{21}(y, x)\right)\right] B_{1}(x)+\operatorname{tr}_{2}\left[\operatorname{tr}_{1}\left(\bar{r}_{12}(x, y) M_{1}(x)\right), M_{2}(y)\right] B_{2}(y)
\end{aligned}
$$

The cyclicity of the traces is used to prove the previous relations. Then, by using (2.20), one concludes the proof.

Remark 3. Note that $t(x)$ and $b(y)$ do not necessarily commute.

\section{FRT PRESENTATION OF FIXED POINT SUBALGEBRAS OF $\widehat{s l_{2}}$}

In this section, based on one of the simplest example of classical Yang-Baxter algebra $\mathscr{T}$ associated with $\widehat{s l_{2}}$, we present four different explicit examples of non-standard classical Yang-Baxter algebra $\mathscr{B}$. Firstly, the Serre-Chevalley and Cartan-Weyl presentations of the affine Kac-Moody algebra $\widehat{s l_{2}}$ are recalled. Secondly, the FRT presentation of $\widehat{s l_{2}}$ is given and the automorphisms of Proposition 2.1 are described, see Corollary 3.1. Finally, using Propositions 2.2 and 2.3 we obtain the FRT presentation for four different homomorphic images of $\mathscr{B}$. They are identified with certain fixed point subalgebras of $\widehat{s l_{2}}$. 
3.1. Presentations of $\widehat{s l_{2}}$. The affine Kac-Moody algebra $\widehat{s l_{2}}$ has been studied extensively in the mathematics and physics literature [K85, GO86] (see also [L93), where two presentations are usually considered. The so-called Serre-Chevalley presentation is generated by $\left\{x_{i}^{+}, x_{i}^{-}, k_{i} \mid i=0,1\right\}$ subject to the commutation relations

$$
\begin{aligned}
& {\left[k_{i}, x_{j}^{ \pm}\right]= \pm a_{i j} x_{j}^{ \pm}, \quad\left[k_{i}, k_{j}\right]=0, \quad\left[x_{i}^{+}, x_{j}^{-}\right]=k_{j} \delta_{i+j},} \\
& {\left[x_{i}^{ \pm},\left[x_{i}^{ \pm},\left[x_{i}^{ \pm}, x_{j}^{ \pm}\right]\right]\right]=0 \quad \text { for } \quad i \neq j,}
\end{aligned}
$$

where $\delta_{i}=0$ if $i \neq 0$ and $\delta_{0}=1$. The element $c=k_{0}+k_{1}$ is central and the entries of the Cartan matrix are given by $a_{00}=a_{11}=2$ and $a_{01}=a_{10}=-2$. Alternatively, the Cartan-Weyl presentation of $\widehat{s l_{2}}$ is generated by $\left\{e_{n}, f_{n}, h_{n}, c \mid n \in \mathbb{Z}\right\}$ subject to the commutation relations

$$
\begin{aligned}
& {\left[e_{n}, e_{m}\right]=\left[f_{n}, f_{m}\right]=0,} \\
& {\left[h_{n}, e_{m}\right]=2 e_{n+m}, \quad\left[h_{n}, f_{m}\right]=-2 f_{n+m},} \\
& {\left[h_{n}, h_{m}\right]=2 \text { cn } \delta_{n+m}, \quad\left[e_{n}, f_{m}\right]=h_{n+m}+c n \delta_{n+m},}
\end{aligned}
$$

where $c$ is central. Recall that the isomorphism from the Serre-Chevalley to the Cartan-Weyl presentation is given, up to automorphism, by (see e.g. [L93]): $k_{1} \mapsto h_{0}, \quad x_{1}^{+} \mapsto e_{0}, \quad x_{1}^{-} \mapsto f_{0}, \quad k_{0} \mapsto-c-h_{0}, \quad x_{0}^{+} \mapsto$ $f_{-1}, \quad x_{0}^{-} \mapsto e_{1}, \quad c \mapsto-c$.

We give now an equivalent presentation of $\widehat{s l_{2}}$ using the generic construction exposed in Section 2.1. In this goal, let us introduce the following classical (traceless) r-matrix for $x \neq 1$ associated with the affine Kac-Moody algebra $\widehat{s l_{2}}$ :

$$
r(x)=\frac{1}{x-1}\left(\begin{array}{cccc}
-\frac{1}{2}(x+1) & 0 & 0 & 0 \\
0 & \frac{1}{2}(x+1) & -2 & 0 \\
0 & -2 x & \frac{1}{2}(x+1) & 0 \\
0 & 0 & 0 & -\frac{1}{2}(x+1)
\end{array}\right)
$$

which satisfies the classical Yang-Baxter equation (2.1) and the following relations

$$
r_{12}(x)=-r_{21}(1 / x)=-r_{12}(1 / x)^{t_{1} t_{2}} .
$$

The affine Kac-Moody algebra $\widehat{s l_{2}}$ admits a third presentation, called an FRT presentation, using the results of the previous section. Namely, by defining:

$$
\begin{aligned}
T^{+}(x) & =\left(\begin{array}{cc}
h_{0} / 2 & 2 f_{0} \\
0 & -h_{0} / 2
\end{array}\right)+\sum_{n \geq 1} x^{n}\left(\begin{array}{cc}
h_{n} & 2 f_{n} \\
2 e_{n} & -h_{n}
\end{array}\right), \\
T^{-}(x) & =\left(\begin{array}{cc}
-h_{0} / 2 & 0 \\
-2 e_{0} & h_{0} / 2
\end{array}\right)+\sum_{n \geq 1} x^{-n}\left(\begin{array}{cc}
-h_{-n} & -2 f_{-n} \\
-2 e_{-n} & h_{-n}
\end{array}\right),
\end{aligned}
$$

the relations given in Definition 2.2 are equivalent to the relations (3.3)-(3.5). Let us remark that the traces of $T^{ \pm}(x)$ vanish.

3.2. Automorphisms of $\widehat{s l_{2}}$ and fixed point subalgebras. We now describe explicitly the automorphisms obtained from Proposition 2.1] in the special case of $\widehat{s l_{2}}$.

Proposition 3.1. Let the r-matrix be defined by (3.6). The only solutions of the equations (2.6) are given by:

$$
U=\left(\begin{array}{cc}
k & 0 \\
0 & -k^{*}
\end{array}\right) \quad \text { for } \epsilon=1 \quad \text { or } \quad U(x)=\left(\begin{array}{cc}
0 & 1 / \sqrt{x} \\
\pm \sqrt{x} & 0
\end{array}\right) \quad \text { for } \epsilon= \pm 1,
$$

where $k, k^{*}$ are non-zero scalar parameters.

Proof. By direct computation.

\footnotetext{
${ }^{4}$ We expect this presentation appears in the literature, although we could not find a reference.
} 
In the following, we study the automorphisms of $\widehat{s l_{2}}$ denoted by $\theta_{1}$ (resp. $\theta_{2}$ ) obtained from (2.7) with the particular solution for (2.6)

$$
U=\left(\begin{array}{cc}
1 & 0 \\
0 & -1
\end{array}\right) \quad\left(\operatorname{resp} . U(x)=\left(\begin{array}{cc}
0 & 1 / \sqrt{x} \\
-\sqrt{x} & 0
\end{array}\right)\right) .
$$

We restrict ourselves to these particular solutions since the subalgebras obtained for the more general cases of $U(x)$ (3.10) are isomorphic to the ones we get from (3.11).

The following corollary is deduced from the explicit form of $T^{ \pm}(x)$ given in (3.8)-(3.9) and relation (2.7):

Corollary 3.1. The action of the automorphisms $\theta_{1}, \theta_{2}$ on the generators in the Cartan-Weyl basis of $\widehat{s_{2}}$ is such that:

$$
\begin{aligned}
& \theta_{1}\left(e_{n}\right)=f_{-n}, \quad \theta_{1}\left(f_{n}\right)=e_{-n}, \quad \theta_{1}\left(h_{n}\right)=-h_{-n} \quad \text { and } \quad \theta_{1}(c)=-c, \\
& \theta_{2}\left(e_{n}\right)=e_{-n+1}, \quad \theta_{2}\left(f_{n}\right)=f_{-n-1}, \quad \theta_{2}\left(h_{n}\right)=h_{-n}+c \delta_{n} \quad \text { and } \quad \theta_{2}(c)=-c .
\end{aligned}
$$

In the Serre-Chevalley basis, these automorphisms are given by $\theta_{1}\left(x_{i}^{ \pm}\right)=x_{i}^{\mp}, \theta_{1}\left(k_{i}\right)=-k_{i}$ and $\theta_{2}\left(x_{i}^{ \pm}\right)=$ $x_{1-i}^{\mp}, \theta_{2}\left(k_{i}\right)=-k_{1-i}$.

Note that solely solving the relations (2.6), two well-known automorphisms of $\widehat{s_{2}}$ are recovered. In the literature, the automorphism $\theta_{1}$ is called the Chevalley involution, whereas the automorphism $\theta_{2}$ is a composition of the outer automorphism of $\widehat{s l_{2}}$ and the Chevalley involution.

According to the choice of automorphisms of $\widehat{s l_{2}}$, an FRT presentation for two different fixed point subalgebras of the affine Kac-Moody algebra $\widehat{s l}_{2}$ can be constructed. First, we consider the fixed point subalgebra $\widehat{s l}_{2}^{\theta_{1}}$. Using (3.8), (3.9), by straightforward simplifications the element (2.12) is written ar 5 :

$$
B(x)=\left(\begin{array}{cc}
0 & \bar{A}_{0} \\
0 & 0
\end{array}\right)+\sum_{n \geq 1} x^{n}\left(\begin{array}{ll}
\bar{G}_{n} & \bar{A}_{-n} \\
\bar{A}_{n} & -\bar{G}_{n}
\end{array}\right) \mapsto T^{+}(x)+\theta_{1}\left(T^{+}(x)\right)=T^{+}(x)+U T^{-}(1 / x)^{t} U^{-1},
$$

where the generators $\bar{A}_{n}, \bar{G}_{n}$ are such that:

$$
\bar{A}_{n} \mapsto 2\left(e_{n}+f_{-n}\right) \quad \text { and } \quad \bar{G}_{n} \mapsto h_{n}-h_{-n} .
$$

Now, consider the second solution of (3.11). For this choice of automorphism, the element $B(x)$ is written as:

$$
\begin{aligned}
B(x) & =\left(\begin{array}{cc}
\bar{K}_{0} / 2 & \bar{Z}_{0}^{-} \\
0 & -\bar{K}_{0} / 2
\end{array}\right)+\sum_{n \geq 1} x^{n}\left(\begin{array}{cc}
\bar{K}_{n} & \bar{Z}_{n}^{-} \\
\bar{Z}_{n}^{+} & -\bar{K}_{n}
\end{array}\right) \\
& \mapsto T^{+}(x)+\theta_{2}\left(T^{+}(x)\right)=T^{+}(x)+U(x) T^{-}(1 / x)^{t} U^{-1}(x)-c x U^{\prime}(x) U(x)^{-1},
\end{aligned}
$$

where the generators $\bar{K}_{n}, \bar{Z}_{n+1}^{+}, \bar{Z}_{n}^{-}$, for $n \in \mathbb{Z}_{\geq 0}$, are such that:

$$
\bar{Z}_{n+1}^{+} \mapsto 2\left(e_{n+1}+e_{-n}\right), \quad \bar{Z}_{n}^{-} \mapsto 2\left(f_{n}+f_{-n-1}\right), \quad \text { and } \quad \bar{K}_{n} \mapsto h_{n}+h_{-n}+c \delta_{n} .
$$

3.3. Classical reflection equation for $\widehat{s l_{2}}$ and fixed point subalgebras. Previously, we identified two different subalgebras of $\mathscr{T}$ as fixed point subalgebras of $\widehat{s l_{2}}$ under the action of certain automorphisms. In this subsection, using Proposition 2.3 we obtain two other known fixed point subalgebras of $\widehat{s l_{2}}$.

Proposition 3.2. Let the r-matrix be defined by (3.6). The most general k-matrix solution of the classical reflection equation 2.14) is given by:

$$
k(x)=\eta(x)\left(\begin{array}{cc}
\alpha(x-1 / x) & \beta+\gamma / x \\
-\beta-\gamma x & \delta(x-1 / x)
\end{array}\right),
$$

where $\alpha, \beta, \gamma$ and $\delta$ are scalar parameters and $\eta(x)$ is an arbitrary function.

Proof. By direct computation.

\footnotetext{
${ }^{5}$ The central charge $c$ does not appear, as the derivative of $U$ is vanishing.
} 
Remark 4. For $\alpha=1, \delta=-1$ and $\beta=\gamma=0$, (resp. $\beta=\gamma$ and $\alpha=\delta=0$ ) the $k$-matrix (3.18) becomes proportional to the matrix $U$ (resp. $U(x)$ ) given in (3.11).

By using Proposition 2.3 with the k-matrix (3.18), a subalgebra of $\widehat{s l_{2}}$ is defined depending on the four parameters $\alpha, \beta, \gamma$ and $\delta$. As examples, we consider the two following choices for $k(x)$.

Example 1. For $\left.k(x)\right|_{\substack{\alpha=\delta=\gamma=0 \\ \beta=\eta(x)=1}}=\left(\begin{array}{cc}0 & 1 \\ -1 & 0\end{array}\right)=\kappa_{+}$, the homomorphism (2.15) becomes

$$
B(x)=\left(\begin{array}{cc}
\bar{H}_{0} / 2 & \bar{F}_{0} / 2 \\
\bar{E}_{0} / 2 & -\bar{H}_{0} / 2
\end{array}\right)+\sum_{n \geq 1} x^{n}\left(\begin{array}{cc}
\bar{H}_{n} & \bar{F}_{n} \\
\bar{E}_{n} & -\bar{H}_{n}
\end{array}\right) \mapsto T^{+}(x)+\kappa_{+} T^{-}(1 / x)^{t} \kappa_{+}^{-1},
$$

where the generators $\bar{H}_{n}, \bar{E}_{n}, \bar{F}_{n}$, for $n \in \mathbb{Z}_{\geq 0}$, are such that:

$$
\bar{E}_{n} \mapsto 2\left(e_{n}+e_{-n}\right) \quad, \quad \bar{F}_{n} \mapsto 2\left(f_{n}+f_{-n}\right) \quad \text { and } \quad \bar{H}_{n} \mapsto h_{n}+h_{-n} .
$$

This subalgebra is denoted by $\widehat{s l}_{2}^{\kappa_{+}}$.

Example 2. For $\left.k(x)\right|_{\substack{\alpha=\delta=\beta=0 \\ \gamma=\eta(x)=1}}=\left(\begin{array}{cc}0 & 1 / x \\ -x & 0\end{array}\right)=\kappa_{-}(x)$, the homomorphism (2.15) becomes

$$
\begin{aligned}
B(x) & =x^{-1}\left(\begin{array}{cc}
0 & \widetilde{F}_{0} / 2 \\
0 & 0
\end{array}\right)+\left(\begin{array}{cc}
\widetilde{H}_{0} / 2 & \widetilde{F}_{1} \\
0 & -\widetilde{H}_{0} / 2
\end{array}\right)+x\left(\begin{array}{cc}
\widetilde{H}_{1} & \widetilde{F}_{2} \\
\widetilde{E}_{0} / 2 & -\widetilde{H}_{1}
\end{array}\right)+\sum_{n \geq 2} x^{n}\left(\begin{array}{cc}
\widetilde{H}_{n} & \widetilde{F}_{n+1} \\
\widetilde{E}_{n-1} & -\widetilde{H}_{n}
\end{array}\right) \\
& \mapsto T^{+}(x)+\kappa_{-}(x) T^{-}(1 / x)^{t} \kappa_{-}(x)^{-1}-c x \kappa_{-}^{\prime}(x) \kappa_{-}(x)^{-1},
\end{aligned}
$$

where the generators $\widetilde{H}_{n}, \widetilde{E}_{n}, \widetilde{F}_{n}$, for $n \in \mathbb{Z}_{\geq 0}$, are such that:

$$
\widetilde{E}_{n} \mapsto 2\left(e_{n+1}+e_{-n+1}\right) \quad, \quad \widetilde{F}_{n} \mapsto 2\left(f_{n-1}+f_{-n-1}\right) \quad \text { and } \quad \widetilde{H}_{n} \mapsto h_{n}+h_{-n}+2 c \delta_{n} .
$$

This subalgebra is denoted by $\widehat{s l}_{2}^{\kappa-}$.

By using the commutation relations (3.3)-(3.5), one finds that both ${\widehat{s l_{2}}}^{\kappa \pm}$ are isomorphic Lie algebras. Note also that ${\widehat{s l_{2}}}^{\kappa_{+}}$(resp. ${\widehat{s l_{2}}}^{\kappa_{-}}$) can be viewed as the fixed point subalgebra of $\widehat{s l}_{2}$ under the action of the Lusztig automorphism in the Cartan-Weyl presentation $e_{n} \mapsto e_{-n}, f_{n} \mapsto f_{-n}, h_{n} \mapsto h_{-n}$ and $c \mapsto-c$ (resp. $e_{n} \mapsto e_{-n+2}, f_{n} \mapsto f_{-n-2}, h_{n} \mapsto h_{-n}+2 c \delta_{n}$ and $\left.c \mapsto-c\right)$. However, let us emphasize that the Lusztig automorphisms cannot be written in the form of Proposition 2.1.

\section{The Onsager algebras And CURREnt Algebras}

In this section, it is shown that the three non-standard classical Yang-Baxter algebras (2.11) associated with (3.14), (3.16) and (3.19) provide an FRT presentation for the Onsager algebra, augmented Onsager algebra and the $s l_{2}$-invariant Onsager algebra, respectively. In each case, the corresponding current presentations are derived. Using the FRT presentation, we also derive the corresponding commutative subalgebras. In particular, this provides a new derivation of the well-known mutually commuting quantities in integrable models generated from the Onsager algebra such as the Ising $\mathrm{O} 44$ ] or superintegrable chiral Potts models GR85, D91. For the augmented Onsager algebra, it gives classical analogs of the mutually commuting quantities constructed in BB12.

4.1. The Onsager algebra revisited. Introduced in the context of mathematical physics on the exact solution of the two-dimensional Ising model O44, the Onsager algebra is known to admit at least two presentations. The first presentation which originates in Onsager's work $\mathrm{O} 44$ is now recalled.

Definition 4.1. The Onsager algebra $\mathscr{O}$ is generated by $\left\{A_{n}, G_{m} \mid n, m \in \mathbb{Z}\right\}$ subject to the following relations:

$$
\begin{aligned}
& {\left[A_{n}, A_{m}\right]=4 G_{n-m},} \\
& {\left[G_{n}, A_{m}\right]=2 A_{n+m}-2 A_{m-n},} \\
& {\left[G_{n}, G_{m}\right]=0 .}
\end{aligned}
$$


As the Lie bracket is anti-symmetric, $\left[A_{0}, A_{n}\right]+\left[A_{n}, A_{0}\right]=0$. From (4.1), note that

$$
G_{-n}+G_{n}=0 \text {. }
$$

Note that a second presentation is given in terms of two generators $A_{0}, A_{1}$ subject to a pair of relations, the so-called Dolan-Grady relations DG82. They read:

$$
\left[A_{0},\left[A_{0},\left[A_{0}, A_{1}\right]\right]\right]=16\left[A_{0}, A_{1}\right], \quad\left[A_{1},\left[A_{1},\left[A_{1}, A_{0}\right]\right]\right]=16\left[A_{1}, A_{0}\right] .
$$

Theorem 1. The non-standard classical Yang-Baxter algebra 2.11) specialized for

$$
B(x)=\left(\begin{array}{cc}
\mathcal{G}(x) & \mathcal{A}^{-}(x) \\
\mathcal{A}^{+}(x) & -\mathcal{G}(x)
\end{array}\right)
$$

with

$$
\mathcal{G}(x)=\sum_{n \geq 1} x^{n} G_{n}, \quad \mathcal{A}^{-}(x)=\sum_{n \geq 0} x^{n} A_{-n}, \quad \mathcal{A}^{+}(x)=\sum_{n \geq 1} x^{n} A_{n}
$$

and the r-matrix given by

$$
\bar{r}_{12}(x, y)=r_{12}(x / y)+U_{1} r_{12}^{t_{1}}(1 /(x y)) U_{1}^{-1} \text { where } U \text { is given by (3.11), }
$$

provides an FRT presentation of the Onsager algebra.

Proof. Inserting (4.6) into (2.11), one has:

$$
\begin{aligned}
& {[\mathcal{G}(x), \mathcal{G}(y)]=0,} \\
& {\left[\mathcal{G}(x), \mathcal{A}^{+}(y)\right]=\frac{2 x\left(1-y^{2}\right)}{(x-y)(x y-1)} \mathcal{A}^{+}(y)+\frac{2 y}{x-y} \mathcal{A}^{+}(x)+\frac{2 x y}{x y-1} \mathcal{A}^{-}(x)} \\
& {\left[\mathcal{G}(x), \mathcal{A}^{-}(y)\right]=\frac{2 x\left(y^{2}-1\right)}{(x-y)(x y-1)} \mathcal{A}^{-}(y)+\frac{2 x}{y-x} \mathcal{A}^{-}(x)+\frac{2}{1-x y} \mathcal{A}^{+}(x)} \\
& {\left[\mathcal{A}^{+}(x), \mathcal{A}^{+}(y)\right]=\frac{4 x y}{1-x y}(\mathcal{G}(x)-\mathcal{G}(y)) \quad, \quad\left[\mathcal{A}^{+}(x), \mathcal{A}^{-}(y)\right]=\frac{4 x}{x-y}(\mathcal{G}(x)-\mathcal{G}(y)),} \\
& {\left[\mathcal{A}^{-}(x), \mathcal{A}^{-}(y)\right]=\frac{4}{x y-1}(\mathcal{G}(x)-\mathcal{G}(y)) .}
\end{aligned}
$$

Define

$$
\mathcal{G}(x)=\sum_{n \geq 1} x^{n} \bar{G}_{n}, \quad \mathcal{A}^{-}(x)=\sum_{n \geq 0} x^{n} \bar{A}_{-n}, \quad \mathcal{A}^{+}(x)=\sum_{n \geq 1} x^{n} \bar{A}_{n} .
$$

Then, we extract from (4.9)-(4.13) the complete set of relations satisfied by the generators $\bar{A}_{n}, \bar{G}_{m}$ for $n, m \geq 1$. Consider (4.12), (4.13). Expanding around $y=0$ both sides of the three equations and identifying the power series, one obtains equivalently:

$$
\begin{aligned}
{\left[\bar{A}_{n}, \bar{A}_{m}\right] } & =4 \operatorname{sign}(n-m) \bar{G}_{|n-m|}, & {\left[\bar{A}_{-n}, \bar{A}_{-m}\right]=4 \operatorname{sign}(m-n) \bar{G}_{|n-m|}, } \\
\left.\bar{A}_{n}, \bar{A}_{-m}\right] & =4 \bar{G}_{n+m}, & {\left[\bar{A}_{n}, \bar{A}_{0}\right]=4 \bar{G}_{n} \quad \text { for any } \quad n, m \geq 1 . }
\end{aligned}
$$

Consider the r.h.s of (4.10). Around $y=0$, one has:

$$
\frac{2 x\left(1-y^{2}\right)}{(x-y)(x y-1)} \mathcal{A}^{+}(y)=-2 \sum_{m=1}^{\infty} \sum_{n=0}^{m-1} x^{-n} y^{m} \bar{A}_{m-n}-2 \sum_{m=1}^{\infty} \sum_{n=1}^{m} x^{n} y^{m} \bar{A}_{m-n}
$$

whereas

$$
\begin{aligned}
\frac{2 y}{x-y} \mathcal{A}^{+}(x)+\frac{2 x y}{x y-1} \mathcal{A}^{-}(x)= & \sum_{n=1}^{\infty} \sum_{m=1}^{\infty} x^{n} y^{m}\left(2 \bar{A}_{n+m}-2 \bar{A}_{m-n}\right) \\
& +2 \sum_{m=1}^{\infty} \sum_{n=0}^{m-1} x^{-n} y^{m} \bar{A}_{m-n}+2 \sum_{m=1}^{\infty} \sum_{n=1}^{m} x^{n} y^{m} \bar{A}_{m-n}
\end{aligned}
$$

Combining all terms together with the l.h.s of (4.10), one gets equivalently:

$$
\left[\bar{G}_{n}, \bar{A}_{m}\right]=2 \bar{A}_{m+n}-2 \bar{A}_{m-n} \quad \text { for any } n, m \geq 1 \text {. }
$$


The similar analysis for (4.11) gives:

$$
\left[\bar{G}_{n}, \bar{A}_{-m}\right]=2 \bar{A}_{-m+n}-2 \bar{A}_{-m-n} \quad \text { and } \quad\left[\bar{G}_{n}, \bar{A}_{0}\right]=2 \bar{A}_{n}-2 \bar{A}_{-n} \quad \text { for any } \quad n, m \geq 1 .
$$

Finally, from (4.9) we immediatly obtain:

$$
\left[\bar{G}_{n}, \bar{G}_{m}\right]=0 \text { for any } n, m \geq 1 \text {. }
$$

It remains to show that the algebra generated by $\bar{A}_{n}, \bar{G}_{m}$ is isomorphic to the Onsager algebra. With the identification

$$
A_{n}=\bar{A}_{n} \quad \text { and } \quad G_{n}=\operatorname{sign}(n) \bar{G}_{|n|} \quad \text { for any } n, m \in \mathbb{Z},
$$

one obtains the defining relations of the Onsager algebra (4.1)-(4.3).

Remark 5. The fixed point subalgebra of the loop algebra of $s l_{2}$ under the action of $\theta_{1}$ (see relations (3.12)) is isomorphic to the Onsager algebra $\mathscr{O}$ [D91, R91. For $\widehat{s l}_{2}^{\theta_{1}}$, the isomorphism is given by (3.15).

Note that the relations (4.9)-(4.13) provide a current presentation for the Onsager algebra.

Using the general results presented in Section 2.3 (see Propositions 2.4 2.5), the generating functions of the elements in the commutative subalgebras of the Onsager algebra are easily derived. On one hand, by Proposition 2.4 we routinely obtain:

Proposition 4.1. The generating function

$$
t^{\text {ons }}(x)=2 \mathcal{G}(x)^{2}+\mathcal{A}^{+}(x) \mathcal{A}^{-}(x)+\mathcal{A}^{-}(x) \mathcal{A}^{+}(x)
$$

is such that $\left[t^{\text {ons }}(x), t^{\text {ons }}(y)\right]=0$ for any $x, y$.

On the other hand, by Proposition 2.5 it follows:

Proposition 4.2. Let $\kappa, \kappa^{*}, \mu$ be arbitrary scalars. The generating function

$$
b^{\text {ons }}(x)=\left(\kappa+\kappa^{*} / x\right) \mathcal{A}^{+}(x)+\left(\kappa+\kappa^{*} x\right) \mathcal{A}^{-}(x)+\mu(1 / x-x) \mathcal{G}(x)
$$

is such that $\left[b^{\text {ons }}(x), b^{\text {ons }}(y)\right]=0$ for any $x, y$.

Proof. First, one shows that

$$
M(x)=\left(\begin{array}{cc}
\mu / x & \kappa+\kappa^{*} / x \\
\kappa+\kappa^{*} x & \mu x
\end{array}\right)
$$

is a solution of the relation (2.20) with the r-matrix given by (4.8). From (2.21), we immediately get (4.17).

Remark 6. Expanding the generating function $b^{\text {ons }}(x)$ in $x$, one produces the well-known mutually commuting quantities of the Onsager algebra $\mathscr{O}$ O44, GR85, D91. For $k=0,1,2, \ldots$, the coefficients of the power series are given by:

$$
I_{k}=\kappa\left(A_{k}+A_{-k}\right)+\kappa^{*}\left(A_{k+1}+A_{-k+1}\right)+\mu\left(G_{k+1}-G_{k-1}\right) .
$$

4.2. The augmented Onsager algebra revisited . The augmented Onsager algebra has been introduced in [BC12] as a classical analog of the augmented tridiagonal algebra of the first kind [T09, page 5-6], also called the augmented $q-$ Onsager algebra in [BB12, BB17].

Definition 4.2. [BC12] The augmented Onsager algebra $\mathscr{O}^{\text {aug }}$ is generated by $\left\{K_{n}, Z_{m}^{ \pm} \mid n, m \in \mathbb{Z}\right\}$ subject to the following relations

$$
\begin{aligned}
& {\left[Z_{n}^{+}, Z_{m}^{+}\right]=\left[Z_{n}^{-}, Z_{m}^{-}\right]=\left[K_{n}, K_{m}\right]=0,} \\
& {\left[Z_{n}^{+}, Z_{m}^{-}\right]=4\left(K_{n+m}+K_{-n+m+1}\right), \quad\left[K_{n}, Z_{m}^{ \pm}\right]= \pm 2\left(Z_{n+m}^{ \pm}+Z_{-n+m}^{ \pm}\right)} \\
& K_{n}-K_{-n}=0, \quad Z_{n}^{+}-Z_{-n+1}^{+}=0 \quad \text { and } \quad Z_{n}^{-}-Z_{-n-1}^{-}=0 .
\end{aligned}
$$


Let us point out that, from the defining relations (4.20)-(4.21), it is possible to show that the three generators $K_{0}, Z_{0}^{ \pm}$satisfy:

$$
\left[Z_{0}^{ \pm},\left[Z_{0}^{ \pm},\left[Z_{0}^{ \pm}, Z_{0}^{\mp}\right]\right]\right]=0, \quad\left[K_{0}, Z_{0}^{ \pm}\right]= \pm 4 Z_{0}^{ \pm} .
$$

These latter relations can be viewed as the classical analog of the relations in [T09, page 5] or [BB12, eqs. $(3.22)]$.

Theorem 2. The non-standard classical Yang-Baxter algebra (2.11) specialized for

$$
B(x)=\left(\begin{array}{cc}
\mathcal{K}(x) & \mathcal{Z}^{-}(x) \\
\mathcal{Z}^{+}(x) & -\mathcal{K}(x)
\end{array}\right)
$$

with

$$
\mathcal{K}(x)=K_{0} / 2+\sum_{n \geq 1} x^{n} K_{n} \quad, \quad \mathcal{Z}^{-}(x)=\sum_{n \geq 0} x^{n} Z_{n}^{-} \quad, \quad \mathcal{Z}^{+}(x)=\sum_{n \geq 1} x^{n} Z_{n}^{+},
$$

and the r-matrix given by

$$
\bar{r}_{12}(x, y)=r_{12}(x / y)+U_{1}(x) r_{12}^{t_{1}}(1 /(x y)) U_{1}(x)^{-1} \quad \text { where } U(x) \text { is given by (3.11), }
$$

provides an FRT presentation of the augmented Onsager algebra.

Proof. Inserting (4.24) into (2.11), one has:

$$
\begin{aligned}
& {[\mathcal{K}(x), \mathcal{K}(y)]=0 \quad, \quad\left[\mathcal{Z}^{+}(x), \mathcal{Z}^{+}(y)\right]=0 \quad, \quad\left[\mathcal{Z}^{-}(x), \mathcal{Z}^{-}(y)\right]=0,} \\
& {\left[\mathcal{K}(x), \mathcal{Z}^{+}(y)\right]=\frac{2 y(x+1)(y-1)}{(x-y)(x y-1)} \mathcal{Z}^{+}(x)-\frac{2 y\left(x^{2}-1\right)}{(x-y)(x y-1)} \mathcal{Z}^{+}(y),} \\
& {\left[\mathcal{K}(x), \mathcal{Z}^{-}(y)\right]=\frac{2 y\left(x^{2}-1\right)}{(x-y)(x y-1)} \mathcal{Z}^{-}(y)-\frac{2 x(x+1)(y-1)}{(x-y)(x y-1)} \mathcal{Z}^{-}(x),} \\
& {\left[\mathcal{Z}^{+}(x), \mathcal{Z}^{-}(y)\right]=\frac{4 x}{(x-y)(x y-1)}((x+1)(y-1) \mathcal{K}(x)-(x-1)(y+1) \mathcal{K}(y)) .}
\end{aligned}
$$

Define

$$
\mathcal{K}(x)=\bar{K}_{0} / 2+\sum_{n \geq 1} x^{n} \bar{K}_{n} \quad, \quad \mathcal{Z}^{-}(x)=\sum_{n \geq 0} x^{n} \bar{Z}_{n}^{-} \quad, \quad \mathcal{Z}^{+}(x)=\sum_{n \geq 1} x^{n} \bar{Z}_{n}^{+} .
$$

Then, we extract from (4.27)-(4.30) the complete set of relations satisfied by the generators $\bar{K}_{n}, \bar{Z}_{n}^{-}, \bar{Z}_{m}^{+}$for $n \geq 0, m \geq 1$. Consider (4.30). Expanding around $y=0$, equivalently we get:

$$
\left[\bar{Z}_{n}^{+}, \bar{Z}_{m}^{-}\right]=4\left(\bar{K}_{n+m}+\bar{K}_{|n-m-1|}\right) \text { for any } n, m \geq 1
$$

Consider (4.28). Around $y=0$, we get:

$$
\begin{array}{ll}
{\left[\bar{K}_{n}, \bar{Z}_{m}^{+}\right]=2\left(\bar{Z}_{n+m}^{+}+\bar{Z}_{n-m+1}^{+}\right)} & \text {for any } \quad 1 \leq m \leq n \\
{\left[\bar{K}_{n}, \bar{Z}_{m}^{+}\right]=2\left(\bar{Z}_{n+m}^{+}+\bar{Z}_{-n+m}^{+}\right) \quad \text { for any } \quad m>n \geq 0 .}
\end{array}
$$

Consider (4.29). Around $y=0$, we get:

$$
\begin{aligned}
& {\left[\bar{K}_{n}, \bar{Z}_{m}^{-}\right]=-2\left(\bar{Z}_{n+m}^{-}+\bar{Z}_{-n+m}^{-}\right) \quad \text { for any } \quad m \geq n \geq 0,} \\
& {\left[\bar{K}_{n}, \bar{Z}_{m}^{-}\right]=-2\left(\bar{Z}_{n+m}^{-}+\bar{Z}_{n-m-1}^{-}\right) \quad \text { for any } \quad 1 \leq m<n,} \\
& {\left[\bar{K}_{n}, \bar{Z}_{0}^{-}\right]=-2\left(\bar{Z}_{n}^{-}+\bar{Z}_{n-1}^{-}\right) \quad \text { for any } n \geq 1 .}
\end{aligned}
$$

Finally, from (4.27) we immediately obtain:

$$
\left[\bar{K}_{n}, \bar{K}_{n^{\prime}}\right]=0, \quad\left[\bar{Z}_{m}^{+}, \bar{Z}_{m^{\prime}}^{+}\right]=0, \quad\left[\bar{Z}_{n}^{-}, \bar{Z}_{n^{\prime}}^{-}\right]=0 \quad \text { for any } n, n^{\prime} \geq 0, m, m^{\prime} \geq 1 .
$$

It remains to show that the algebra generated by $\bar{K}_{n}, \bar{Z}_{m}^{ \pm}$is isomorphic to the augmented Onsager algebra. With the identification

$$
\begin{array}{llllll}
K_{n}=\bar{K}_{|n|}, & Z_{n}^{+}=\bar{Z}_{n}^{+} \quad \text { if } \quad n \geq 1, \quad Z_{n}^{+}=\bar{Z}_{-n+1}^{+} \quad \text { if } \quad n \leq 0, \\
Z_{n}^{-}=\bar{Z}_{n}^{-} \quad \text { if } \quad n \geq 0, \quad Z_{n}^{-}=\bar{Z}_{-n-1}^{-} \quad \text { if } \quad n<0,
\end{array}
$$

one obtains the defining relations of the augmented Onsager algebra (4.20)-(4.21). 
Corollary 4.1. The augmented Onsager algebra is isomorphic to the fixed point subalgebra $\widehat{s l}_{2}^{\theta_{2}}$ generated by (3.17).

Note that the relations (4.27)-(4.30) provide a current presentation for the augmented Onsager algebra.

Generating functions of elements in the commutative subalgebras of the augmented Onsager algebra are now derived. By Propositions 2.4 we routinely obtain:

Proposition 4.3. The generating function

$$
t^{a u g}(x)=2 \mathcal{K}(x)^{2}+\mathcal{Z}^{+}(x) \mathcal{Z}^{-}(x)+\mathcal{Z}^{-}(x) \mathcal{Z}^{+}(x)
$$

is such that $\left[t^{\text {aug }}(x), t^{\text {aug }}(y)\right]=0$ for any $x, y$.

On the other hand, by Proposition 2.5 it follows:

Proposition 4.4. Let $\tau, \nu, \nu^{*}$ be arbitrary scalars. The generating function

$$
b^{\text {aug }}(x)=\tau \mathcal{K}(x)+\nu(1+1 / x) \mathcal{Z}^{+}(x)+\nu^{*}(x+1) \mathcal{Z}^{-}(x)
$$

is such that $\left[b^{\text {aug }}(x), b^{\text {aug }}(y)\right]=0$ for any $x, y$.

Proof. One shows that

$$
M(x)=\left(\begin{array}{cc}
\tau & \nu(1+1 / x) \\
\nu^{*}(x+1) & 0
\end{array}\right)
$$

is a solution of the relation (2.20) with the r-matrix (4.26). From (2.21), we immediately get (4.33).

Remark 7. Expanding the generating function $b^{a u g}(x)$ in $x$, one produces mutually commuting quantities of the augmented Onsager algebra $\mathscr{O}^{\text {aug }}$. For $k=0,1,2, \ldots$ and the convention $Z_{0}^{+}=Z_{-1}^{-}=0$, the coefficients of the power series read:

$$
I_{k}^{a u g}=\tau K_{k}+\nu\left(Z_{k}^{+}+Z_{k+1}^{+}\right)+\nu^{*}\left(Z_{k-1}^{-}+Z_{k}^{-}\right) .
$$

4.3. The $s l_{2}$-invariant Onsager algebra. In this subsection, we introduce an algebra that we call the $s l_{2}$ invariant algebra. This algebra is viewed as the classical analog of the $U_{q}\left(g l_{2}\right)$-invariant $q$-Onsager algebra introduced in [BB17, subsection 2.4].

Definition 4.3. The $s_{2}$-invariant Onsager algebra $\mathscr{O}^{i n v}$ is generated by $\left\{H_{n}, E_{n}, F_{n} \mid n \in \mathbb{Z}\right\}$ subject to the following relations

$$
\begin{aligned}
& {\left[E_{n}, E_{m}\right]=\left[F_{n}, F_{m}\right]=\left[H_{n}, H_{m}\right]=0,} \\
& {\left[H_{n}, E_{m}\right]=2\left(E_{n+m}+E_{-n+m}\right), \quad\left[H_{n}, F_{m}\right]=-2\left(F_{n+m}+F_{-n+m}\right),} \\
& {\left[E_{n}, F_{m}\right]=H_{n+m}+H_{-n+m} .}
\end{aligned}
$$

As a consequence of the commutation relations (4.36)-(4.38), linear relations among the generators $\left\{E_{n}, F_{n}, H_{n} \mid n \in \mathbb{Z}\right\}$ can be exhibited. Define:

$$
\mathbb{E}_{n}=E_{n}-E_{-n}, \quad \mathbb{F}_{n}=F_{n}-F_{-n}, \quad \mathbb{H}_{n}=H_{n}-H_{-n} .
$$

From (4.38) and (4.36) one gets

$$
\left[F_{n}, \mathbb{E}_{m}\right]=\left[E_{n}, \mathbb{F}_{m}\right]=\left[E_{n}, \mathbb{E}_{m}\right]=\left[F_{n}, \mathbb{F}_{m}\right]=0 .
$$

Using (4.38) with $n=0$ we have $H_{m}=\frac{1}{2}\left[E_{0}, F_{m}\right]$. Applying the Jacobi identify we obtain

$$
\left[H_{n}, \mathbb{E}_{m}\right]=\frac{1}{2}\left[\left[E_{0}, F_{m}\right], \mathbb{E}_{m}\right]=0, \quad\left[H_{n}, \mathbb{F}_{m}\right]=\frac{1}{2}\left[\left[E_{0}, F_{m}\right], \mathbb{F}_{m}\right]=0 .
$$

Also, using the commutation relations (4.37), we find

$$
\left[\mathbb{H}_{n}, F_{m}\right]=\left[\mathbb{H}_{n}, E_{m}\right]=\left[\mathbb{H}_{n}, H_{m}\right]=0 .
$$

\footnotetext{
${ }^{6}$ In the context of the half-infinite XXZ spin chain, the $U_{q}\left(g l_{2}\right)$-invariant $q$-Onsager algebra algebra characterizes the hidden non-Abelian infinite dimensional symmetry of the Hamiltonian with $U_{q}\left(g l_{2}\right)$-invariant (special diagonal) boundary conditions (for the finite open XXZ spin chain, see [PS90]). This motivates the terminology used in [BB17] and here. In the defining relations of the $s l_{2}$-invariant algebra, one recognizes the $s l_{2}$-subalgebra 4.45].
} 
Thus, according to (4.40), (4.41) and (4.42) the elements $\left\{\mathbb{H}_{n}, \mathbb{E}_{n}, \mathbb{F}_{n}\right\}$ with $n \in \mathbb{Z}$ belong to the center of $\mathscr{O}^{i n v}$. Finally, observe that the commutation relations (4.37) imply

$$
\left[H_{n}, \mathbb{F}_{m}\right]=-2\left(\mathbb{F}_{n+m}+\mathbb{F}_{-n+m}\right), \quad\left[H_{n}, \mathbb{E}_{m}\right]=2\left(\mathbb{E}_{n+m}+\mathbb{E}_{-n+m}\right) .
$$

Together with (4.41), it follows $\mathbb{F}_{n+m}+\mathbb{F}_{-n+m}=\mathbb{E}_{n+m}+\mathbb{E}_{-n+m}=0$ for all $n$ and $m$. In particular, for $n=0$ one gets $\mathbb{E}_{m}=\mathbb{F}_{m}=0$. It implies $\mathbb{H}_{m}=\frac{1}{2}\left[E_{0}, \mathbb{F}_{m}\right]=0$. As a consequence, in the algebra $\mathscr{O}^{\text {inv }}$ the following linear relations hold:

$$
E_{n}-E_{-n}=0, \quad F_{n}-F_{-n}=0, \quad H_{n}-H_{-n}=0 .
$$

From the defining relations (4.36)-(4.38), one finds that the six generators $H_{0}, E_{0}, F_{0}, H_{1}, E_{1}, F_{1}$ satisfy the relations:

$$
\begin{aligned}
& {\left[H_{0}, E_{0}\right]=4 E_{0}, \quad\left[H_{0}, F_{0}\right]=-4 F_{0}, \quad\left[E_{0}, F_{0}\right]=2 H_{0},} \\
& {\left[H_{0}, E_{1}\right]=\left[H_{1}, E_{0}\right]=4 E_{1}, \quad\left[H_{0}, F_{1}\right]=\left[H_{1}, F_{0}\right]=-4 F_{1}, \quad\left[E_{0}, F_{1}\right]=\left[E_{1}, F_{0}\right]=2 H_{1},} \\
& {\left[H_{1},\left[E_{1}, F_{1}\right]\right]=0 .}
\end{aligned}
$$

Note that these relations are the classical analogs of [BB17, eqs. (2.18)].

Theorem 3. The non-standard classical Yang-Baxter algebra (2.11) specialized for

$$
B(x)=\left(\begin{array}{cc}
\mathcal{H}(x) & \mathcal{F}(x) \\
\mathcal{E}(x) & -\mathcal{H}(x)
\end{array}\right)
$$

with

$$
\mathcal{H}(x)=H_{0} / 2+\sum_{n \geq 1} x^{n} H_{n} \quad, \quad \mathcal{E}(x)=E_{0} / 2+\sum_{n \geq 1} x^{n} E_{n} \quad, \quad \mathcal{F}(x)=F_{0} / 2+\sum_{n \geq 1} x^{n} F_{n},
$$

and the r-matrix given by

$$
\bar{r}_{12}(x, y)=r_{12}(x / y)+\kappa_{+} r_{12}^{t_{1}}(1 /(x y)) \kappa_{+}^{-1} \text { where } \kappa_{+} \text {is given in Example } 1 \text {, }
$$

provides an FRT presentation of the sl $_{2}$-invariant Onsager algebra.

Proof. Inserting (4.48) into (2.11), one has:

$$
\begin{aligned}
& {[\mathcal{H}(x), \mathcal{H}(y)]=0 \quad, \quad[\mathcal{E}(x), \mathcal{E}(y)]=0 \quad, \quad[\mathcal{F}(x), \mathcal{F}(y)]=0,} \\
& {[\mathcal{H}(x), \mathcal{E}(y)]=\frac{2}{(x-y)(x y-1)}\left(x\left(y^{2}-1\right) \mathcal{E}(x)-y\left(x^{2}-1\right) \mathcal{E}(y)\right),} \\
& {[\mathcal{H}(x), \mathcal{F}(y)]=-\frac{2}{(x-y)(x y-1)}\left(x\left(y^{2}-1\right) \mathcal{F}(x)-y\left(x^{2}-1\right) \mathcal{F}(y)\right),} \\
& {[\mathcal{E}(x), \mathcal{F}(y)]=\frac{4}{(x-y)(x y-1)}\left(x\left(y^{2}-1\right) \mathcal{H}(x)-y\left(x^{2}-1\right) \mathcal{H}(y)\right) .}
\end{aligned}
$$

Define

$$
\mathcal{H}(x)=\bar{H}_{0} / 2+\sum_{n \geq 1} x^{n} \bar{H}_{n} \quad, \quad \mathcal{E}(x)=\bar{E}_{0} / 2+\sum_{n \geq 1} x^{n} \bar{E}_{n} \quad, \quad \mathcal{F}(x)=\bar{F}_{0} / 2+\sum_{n \geq 1} x^{n} \bar{F}_{n} .
$$

As we proceed by analogy with the previous cases, we omit the details. With the identification

$$
H_{n}=\bar{H}_{|n|}, \quad E_{n}=\bar{E}_{|n|}, \quad F_{n}=\bar{F}_{|n|},
$$

one obtains the defining relations of the $s l_{2}$-invariant Onsager algebra (4.36), (4.38).

Corollary 4.2. The $s_{2}$-invariant Onsager algebra is isomorphic to the fixed point subalgebra $\widehat{s l}_{2}{ }^{\kappa_{+}}$generated by (3.20).

Note that the relations (4.51)-(4.54) provide a current presentation for the Onsager algebra.

Generating functions of elements in the commutative subalgebras of the $s l_{2}$-invariant Onsager algebra are now derived. By Propositions 2.4

Proposition 4.5. The generating function

$$
t^{i n v}(x)=2 \mathcal{H}(x)^{2}+\mathcal{E}(x) \mathcal{F}(x)+\mathcal{F}(x) \mathcal{E}(x)
$$

is such that $\left[t^{i n v}(x), t^{i n v}(y)\right]=0$ for any $x, y$. 
By Proposition 2.5, it follows:

Proposition 4.6. Let $\mu_{0}, \mu_{1}, \mu_{2}$ be arbitrary scalars. The generating function

$$
b^{i n v}(x)=\mu_{0} \mathcal{H}(x)+\mu_{1} \mathcal{E}(x)+\mu_{2} \mathcal{F}(x)
$$

is such that $\left[b^{i n v}(x), b^{i n v}(y)\right]=0$ for any $x, y$.

Proof. One shows that

$$
M(x)=\left(\begin{array}{cc}
\mu_{0} & \mu_{1} \\
\mu_{2} & 0
\end{array}\right)
$$

is a solution of the relation (2.20) for the r-matrix (4.50). From (2.21), we immediately get (4.57).

\section{Concluding Remarks}

In this letter, it is shown that the classical Onsager, augmented Onsager and $s l_{2}$-invariant Onsager algebras fit into the framework of the non-standard classical Yang-Baxter algebras. Using this framework, an FRT presentation is identified in each case. A current presentation is obtained and the generating functions for elements in their commutative subalgebras are constructed.

Here, we mainly focused on different types of non-standard classical Yang-Baxter algebras associated with one of the simplest affine Kac-Moody algebra $\widehat{s l_{2}}$, and their explicit relations with the Onsager algebras. However, it is clear that the framework proposed in Section 2 can be easily applied to any higher rank affine Lie algebra and their fixed point subalgebras. Although not discussed in this letter, an FRT presentation and associated current presentations for any of the higher rank classical Onsager algebras [UI95, BB09] could be derived in this framework. Another generalization of the construction presented here would be to consider automorphisms of order greater than 2. Indeed, such automorphisms have been studied for the rational r-matrix in CY07, and provide interesting integrable systems.

In view of the recently proposed presentation of the $q$-Onsager algebra in terms of root vectors BK17. satisfying a $q$-deformed analog of the Onsager's relations (4.1)-(4.3), it is tempting to reconsider the connection between the quantum reflection algebra and coideal subalgebras of $U_{q}\left(\widehat{s l_{2}}\right)$ [MRS03, or the $q-$ Onsager algebra [B04, BK05] in light of the results presented here. Besides the two Examples 10 2, it is also natural to ask for an interpretation of the homomorphic image (2.15) in terms of $\widehat{s l_{2}}$ subalgebras for the the most general solution (3.18) of the classical reflection equation (2.14).

From the point of view of applications to quantum integrable systems, the FRT presentation for the Onsager algebras and related current presentations offer a different perspective for the analysis of wellknown integrable models such as the Ising or superintegrable Potts model. For instance, by analogy with the analysis of [BB12], a free field realization of the Onsager's currents may be considered to solve the spectral problem for the mutually commuting quantities (4.17) and study correlation functions of local fields. In particular, it would be nice if it could provide a derivation of the celebrated Painlevé equations [P80, MPW81, FZ03] for the Ising model based on the Onsager algebra.

Some of these problems will be considered elsewhere.

Acknowledgements: We thank an anonymous referee for comments. We thank Hubert Saleur and Jasper Stokman for discussions, which motivated the construction of an FRT presentation for the Onsager algebra and its current presentation that could extend to any higher rank cases. P.B. and N.C. are supported by C.N.R.S. S.B. and N.C. thanks the LMPT for hospitality, where part of this work has been done. S.B. is supported by a public grant as part of the Investissement d'avenir project, reference ANR-11-LABX-0056LMH, LabEx LMH.

\section{REFERENCES}

[B04] P. Baseilhac, An integrable structure related with tridiagonal algebras, Nucl.Phys. B 705 (2005) 605-619, arXiv:math-ph/0408025.

[BB09] P. Baseilhac and S. Belliard, Generalized q-Onsager algebras and boundary affine Toda field theories, Lett. Math. Phys. 93 (2010) 213-228, arXiv:0906.1215. 
[BB17] P. Baseilhac and S. Belliard, An attractive basis for the $q-$ Onsager algebra, arXiv:1704.02950.

[BB12] P. Baseilhac and S. Belliard, The half-infinite XXZ chain in Onsager's approach, Nucl. Phys. B 873 (2013) 550-583, arXiv: 1211.6304

[BB17] P. Baseilhac and S. Belliard, Non-Abelian symmetries of the half-infinite XXZ spin chain, Nucl. Phys. B 916 (2017) 373-385, arXiv: 1611.05390

[BK05] P. Baseilhac and K. Koizumi, A new (in)finite dimensional algebra for quantum integrable models, Nucl. Phys. B 720 (2005) 325-347, arXiv:math-ph/0503036

[BK17] P. Baseilhac and S. Kolb, Braid group action and root vectors for the q-Onsager algebra, arXiv:1706.08747.

[BS09] P. Baseilhac and K. Shigechi, A new current algebra and the reflection equation, Lett. Math. Phys. 92 (2010) 47-65, arXiv:0906.1482

[BD82] A.A. Belavin and V.G. Drinfel'd, Solutions of the classical Yang-Baxter equation for simple Lie algebras, Funct. Anal. Appl. 16 (1982) 159.

[BC12] S. Belliard and N. Crampe, Coideal algebras from twisted Manin triple, J. Geo. Phys. 62 (2012) 2009-2023, arXiv: 1202.2312 .

[CRT04] A. Chervov, L. Rybnikov and D. Talalaev, Rational Lax operators and their quantization, ITEP-TH-105/03, arXiv:hep-th/0404106

D. Talalaev, Quantization of the Gaudin System, ITEP-TH-14/04, arXiv:hep-th/0404153

[CY07] N. Crampe and C.A.S. Young, Integrable Models From Twisted Half Loop Algebras, J. Phys. A 40 (2007) 5491-5510, math-ph/0609057

[D91] B. Davies, Onsager's algebra and superintegrability, J. Phys. A 23 (1990) 2245-2261; B. Davies, Onsager's algebra and the Dolan-Grady condition in the non-self-dual case, J. Math. Phys. 32 (1991) $2945-2950$.

[DG82] L. Dolan and M. Grady, Conserved charges from self-duality, Phys. Rev. D 25 (1982) $1587-1604$.

[D83] V.G. Drinfel'd, Hamiltonian structures on Lie groups, Lie bialgebras, and the geometrical meaning of the Yang-Baxter equations, Dokl. Akad, Nauk SSSR, 268 (1983) 285.

[FRT87] L.D. Faddeev, N.Y. Reshetikhin and L.A. Takhtajan, Quantization of Lie groups and Lie algebras, LOMI preprint, Leningrad, (1987) ; Leningrad Math. J. 1 (1990) 193.

[FZ03] P. Fonseca and A. Zamolodchikov, Ward Identities and Integrable Differential Equations in the Ising Field Theory, RUNHETC-2003-28, arXiv:hep-th/0309228

[G83] M. Gaudin, Diagonalisation d'une classe d'Hamiltoniens de spin, J. Phys. 37 (1976) 1087; La Fonction d'onde de Bethe, Paris: Masson (1983).

[GR85] G. von Gehlen and V. Rittenberg, $\mathbb{Z}_{n}$-symmetric quantum chains with infinite set of conserved charges and $\mathbb{Z}_{n}$ zero modes, Nucl. Phys. B 257 (1985) 351.

[GO86] P. Goddard and D. Olive, Kac-Moody and Virasoro algebras in relation to quantum physics, Int. Jour. Mod. Phys. A 1 (1986) 303.

[H95] K. Hikami, Separation of Variables in BC-type Gaudin Magnet, solv-int/9506001, J. Phys. A. 28 (1995) 4053.

[HKW92] K. Hikami, P.P. Kulish and M Wadati, Construction of Integrable Spin Systems with Long-Range Interactions, J. Phys. Soc. Jpn. 61 (1992) 3071.

[IT09] T. Ito and P. Terwilliger, The augmented tridiagonal algebra, Kyushu Journal of Mathematics 64 (2010) 81-144, arXiv:0904.2889. 1 .

[J89] B. Jurčo, Classical Yang-Baxter equations and quantum integrable systems, J. Math. Phys 30 (1989) 1289.

[K85] V.G. Kac, Infinite Dimensional Lie Algebras, Cambridge University Press (1985).

[K12] S. Kolb, Quantum symmetric Kac-Moody pairs, Adv. Math. 267 (2014), 395-469, arXiv:1207.6036r1.

[L93] A. LeClair, Spectrum generating affine Lie algebras in massive field theory, Nucl. Phys. B 415 (1994) 734-780, arXiv:hep-th/9305110

[MPW81] B.M. McCoy, J.H.H. Perk and T.T. Wu, Ising field theory: quadratic difference equations for the n-point Green's functions on the lattice, Phys. Rev. Lett. 46 (1981) 757.

[MRS03] A. Molev, E. Ragoucy and P. Sorba, Coideal subalgebras in quantum affine algebras, Rev. Math. Phys 15 (2003) 789822, arXiv:math/0208140.

[O44] L. Onsager, Crystal Statistics. I. A Two-Dimensional Model with an Order-Disorder Transition, Phys. Rev. 65 (1944) $117-149$.

[PS90] V. Pasquier and H. Saleur, Common structures between finite systems and conformal field theories through quantum groups, Nucl. Phys. B 330 (1990) 523-556.

[P80] J.H.H. Perk, Quadratic identities for the Ising model correlations, Phys. Lett. A 79 (1980) 3-5.

[P87] J.H.H. Perk, Star-triangle equations, quantum Lax pairs, and higher genus curves, Theta FunctionsBowdoin 1987, Part 1 Leon Ehrenpreis and Robert C. Gunning, Editors; The Early History of the Integrable Chiral Potts Model and the Odd-Even Problem, arXiv:1511.08526

[RS90] N.Yu. Reshetikhin and M.A. Semenov-Tian-Shansky, Central extensions of quantum current groups, Lett. Math. Phys. 19 (1990) 133-142.

[R91] S.S. Roan, Onsager algebra, loop algebra and chiral Potts model, MPI 91-70, Max-Planck- Institut fur Mathematik, Bonn (1991).

[S83] M. Semenov Tian Shansky, What is a classical r-matrix ?, Funct. Anal. and its Appl. 17 (1983) $259-272$.

[S87] E.K. Sklyanin, Boundary conditions for integrable equations, Funct. Anal. and its Appl. 21 (1987) 8687.

[S88] E.K. Sklyanin, Boundary conditions for integrable quantum systems, J. Phys. A 21 (1988) $2375-2389$.

[S80] E.K. Sklyanin, On complete integrability of the LandauLifshitz equation, Preprint LOMI, E-3-79, Leningrad, LOMI (1980); The quantum inverse scattering method, Zap. Nauchn.Sem. LOMI 95 (1980) 55. 
[Sk06a] T. Skrypnyk, Integrable quantum spin chains, non-skew symmetric r-matrices and quasigraded Lie algebras, J. Geom. Phys. 57 (2006) 53;

Quantum integrable systems, non-skew-symmetric r-matrices and algebraic Bethe ansatz, J. Math. Phys. 48 (2007) 023506 ; Generalized Gaudin spin chains, nonskew symmetric r-matrices, and reflection equation algebras, J. Math. Phys. 48 (2007) 113521 ;

Generalized Gaudin systems in a magnetic field and non-skew-symmetric r-matrices, J. Phys. A 40 (2007) 13337;

Non-skew-symmetric classical r-matrices and integrable cases of the reduced BCS model, J. Phys. A 42 (2009) 472004.

[Sk06b] T. Skrypnyk, Generalized quantum Gaudin spin chains, involutive automorphisms and twisted classical r-matrices, J. Math. Phys. 47 (2006) 033511.

[T99] P. Terwilliger, Two relations that generalize the $q$-Serre relations and the Dolan-Grady relations, Proceedings of the Nagoya 1999 International workshop on physics and combinatorics. Editors A. N. Kirillov, A. Tsuchiya, H. Umemura. 377-398, math.QA/0307016

[U195] D. Uglov and L. Ivanov, sl(N) Onsager's algebra and integrability, J. Stat. Phys. 82 (1996) 87, arXiv:hep-th/9502068v1.

* Laboratoire de Mathématiques et Physique Théorique CNRS/UmR 7350, Fédération Denis Poisson FR2964, Université de Tours, Parc de Grammont, 37200 Tours, FRAnCE

E-mail address: baseilha@lmpt.univ-tours.fr

** Institut de Physique Théorique, DSM, CEA, URA2306 CNRS Saclay, F-91191 Gif-sur-Yvette, FRANCE

E-mail address: samuel.belliard@gmail.com

$\dagger$ Laboratoire Charles Coulomb (L2C), UMR 5221 CNRS-Université de Montpellier, Montpellier, F-France

E-mail address: nicolas.crampe@umontpellier.fr 